\title{
Rotational Coupling of the Pinned Core Superfluid
}

\author{
M. Jahan-Miri \\ Department of Physics, Shiraz University, Shiraz 71454, Iran \\ jahan@physics.susc.ac.ir
}

\begin{abstract}
The effects of pinning between fluxoids and vortices in the core of a neutron star, on the dynamics of the core neutron superfluid are considered. The pinning impedes, but does not absolutely block, any radial as well as azimuthal motion of the neutron vortices with respect to the lattice of fluxoids. The time scale for the coupling of rotation of the core superfluid to the rest of the star is calculated, allowing for the effect of the finite frictional force on the neutron vortices due to their pinning with the fluxoids. This turns out to be the dominant mechanism for the coupling of the core of a neutron star to its crust, as compared to the role of electron scattering, for most cases of interest. Furthermore, different behaviors for the post-glitch response of the core superfluid are distinguished that might be tested against the relevant observational data. Also, a conceptually important case (and controversial too, in the earlier studies on the role of the crustal superfluid) is realized where a superfluid may remain decoupled in spite of an spinning up of its vortices.
\end{abstract}

Subject headings: stars: neutron - hydrodynamics - pulsars

\section{INTRODUCTION}

The neutron superfluid in the core of a neutron star coexists with the super-conducting protons and the "normal" degenerate electrons. The interior charged plasma including the lattice of the proton vortices (fluxoids) is expected to be strongly coupled to the lattice of nuclei and the electrons in the crust due to the strong magnetic field present, with a coupling time scale $\leq 10 \mathrm{~s}$ (Alpar et al. 1984b). All charged components of the star are hence considered as one co-rotating "component", referred to as the "crust"; in contrast to the neutron superfluid in the core, being the second "component". An isolated neutron star is subject to an electromagnetic spin-down torque that acts on its magnetic field, hence on the "crust". The core superfluid would be likewise driven to follow the long-term spinning down 
of the crust through the mutual coupling mechanism that would operate between the crust and the superfluid vortices. The force, on the vortices, responsible for such a spinning down (or any assumed short-term relaxation) of the core superfluid has been, generally, considered to be only that of the scattering of the electrons off the vortex cores. The dominant scattering effect is that of the induced magnetization of the vortices caused by a "drag" between the proton and neutron condensates. The associated vortex velocity relaxation time scale is $\tau_{\mathrm{e}} \sim 10 P_{\mathrm{s}}-20 P_{\mathrm{s}}$, where $P_{\mathrm{s}}$ is the spin period of the star (Alpar et al. 1984a).

However, in the quantum liquid interior of a neutron star a neutron vortex is expected to "pin" to a fluxoid should the two structures overlap. The mechanism of the pinning is associated with either the proton density perturbations or the magnetized nature of both the fluxoids as well as the neutron vortices. The strength $E_{\mathrm{P}}$ of the corresponding energy barrier has been estimated as $E_{\mathrm{P}} \sim 0.1$ or $1.0 \mathrm{MeV}$, for the two mechanisms, respectively. Likewise, the effective length scale $d_{\mathrm{P}}$ of the pinning interaction is expected to be of the order of the coherence length $\xi_{\mathrm{p}}$ or the London penetration depth $\lambda_{\mathrm{p}}$ of the proton superconductor for the above two mechanisms, respectively. The effective pinning force (per intersection) $f_{\mathrm{P}}=\frac{E_{\mathrm{P}}}{d_{\mathrm{P}}}$ is therefore roughly the same for the two interaction mechanisms, since the value of $E_{\mathrm{P}}$ due to the magnetic interaction is larger than that of the density perturbation by the same ratio as the inverse of their corresponding $d_{\mathrm{P}}$ values, ie. $\frac{\lambda_{\mathrm{p}}}{\xi_{\mathrm{p}}} \sim 10$ (Muslimov \& Tsygan 1985; Sauls 1989; Jones 1991a, 2006).

The consequences of such a pinning with respect to the radial motion of the vortices have been discussed in the literature, to some extent. The outward moving vortices in a slowing down neutron star have been argued to sweep the fluxoids along with them and hence expel the magnetic flux out of the stellar core (Srinivasan et al. 1990; Jones 1991b; Sedrakian \& Sedrakian 1992). The magnetic evolution of pulsars, particularly those in binary systems, has been calculated, based on this effect (Ding et al. 1993; Jahan-Miri \& Bhattacharya 1994; Jahan-Miri 1996; Urpin et al 1997; Jahan-Miri 2000). Also, due to the steady-state slowing down of the star, the superfluid would be rotating faster than the vortices, resulting in a radial Magnus "force" that drives them against the pinning barriers. Glitch inducing mechanisms, caused by the core superfluid, have been suggested on this ground, invoking the possibility of sudden disturbances (Muslimov \& Tsygan 1985), and based on the predicted long-term evolution of the steady-state value of the associated rotational lag between the superfluid and the vortices (Jahan-Miri 2002). Also, the expected post-glitch behavior of the observable rotation frequency of the crust of a neutron star has been discussed in this context (Chau et al. 1992).

The pinning would however also impede any assumed relative azimuthal motion between the two lattices of the vortex lines, and impart azimuthal forces. That is, it could impart 
a torque, and act as a means for a transfer of angular momentum to the core superfluid. The effect is, in principle, same as the role of the standard "static" frictional force, realized in the laboratory experiments on a rotating superfluid, in presence of the pinning centers (Tsakadze \& Tsakadze 1980; Adams et al. 1985). Such a role for the azimuthal component of the pinning force in the core of a neutron star was referred to by Jahan-Miri (1998), and has been recently studied by Sidery \& Alpar (2009). These authors assume however absolute pinning, and assign an infinite strength to the azimuthal component of the pinning force, allowing only for a sliding motion of the vortices along the floxoids, as was first suggested by Sauls (1989). It should be clarified, at the outset, that we are concerned with only an application of the standard results and basic formulation of the superfluid rotational dynamics, at the hydrodynamical level (Sonin 1987), avoiding complications due to the microscopic details. We adopt the generally accepted standard structure for the interior of a neutron star. We attempt to include the role of the azimuthal force on the vortices, due to their pinning with the fluxoids, in the rotational dynamics of the core superfluid, in addition to the viscous drag force, due to the scattering of the electrons.

In the following we discuss the rotational dynamics of neutron stars taking into account also the above effects (both radial, as well as azimuthal) due to the pinning of the vortices in the core of the star. The steady-state relative rotation of the different components of a neutron star is briefly described, distinguishing between the cases of pinned and unpinned vortices in the core superfluid. It may be noted, that a clear picture of the steady-state relative rotations is indeed needed in order to follow the discussion further. The vortexvelocity relaxation time scale is then calculated, allowing also for the finite effect of the frictional force of the pinning with the fluxoids. The dynamical timescale for the coupling of the pinned core superfluid is thence derived. In addition, qualitatively different behaviors for the response of the core superfluid to a jump in the rotation frequency of the "crust" are distinguished, depending on the magnitude of the jump, in the case of pinned superfluid. In the final section, the relevance of our results to the observational data on pulsars glitches

are discussed briefly, indicating the possibility of distinguishing among the different cases considered.

\section{The CORE SUPERFLUID}

Superfluid vortices move with the local superfluid velocity except when there is an external force acting on a vortex. For a given external force $F_{\text {ex }}$, per unit length of the vortex, the equation of motion is given (Sonin 1987) as

$$
\vec{F}_{\mathrm{ex}}=\rho_{\mathrm{s}} \vec{\kappa} \times\left(\vec{v}_{\mathrm{s}}-\vec{v}_{\mathrm{L}}\right)
$$


where $\overrightarrow{v_{\mathrm{S}}}$ and $\overrightarrow{v_{\mathrm{L}}}$ are velocities of the local superfluid and the vortex line, respectively, $\rho_{\mathrm{s}}$ is the superfluid mass density, $\vec{\kappa}$ is the vorticity of the vortex line directed along the rotation axis, with a magnitude $\kappa=\frac{h}{2 m_{\mathrm{n}}}$ for the neutron superfluid, where $m_{\mathrm{n}}$ is the mass of a neutron. The kinematic side of the equation is generally referred to as the Magnus "force". The force, arising from the gradient of the superfluid kinetic energy, is exerted between the superfluid and the vortices, and explains how a "mutual" friction between the superfluid and its environment is realized. As is usual in this field, we will neglect the collective effects of the vortex lattice, finite tension of a vortex, as well as any relevant non-dissipative force on a vortex by the lattice, which has been studied recently for the vortices in the crust of neutron stars (Link 2009).

In the case of a rotating superfluid, the number density $n_{\mathrm{v}}$, per unit area, of the vortices obeys $\kappa n_{\mathrm{v}}=2 \Omega_{\mathrm{s}}$, and the rate $\dot{\Omega}_{\mathrm{s}}$ of change of the rotation frequency $\Omega_{\mathrm{s}}$ of the superfluid is

associated with a radial velocity $v_{r}$ of the vortices, $\dot{\Omega}_{\mathrm{s}}=-2 \frac{\Omega_{\mathrm{s}}}{r} v_{r}$, where $r$ is the distance from the rotation axis, and $v_{r}>0$ is in the outward direction. Thus, a spinning down (up) torque on a rotating superfluid is accompanied by a radial outward (inward) motion of the vortices.

\subsection{Steady-State Relative Rotations}

During the steady state spinning down of a neutron star, the vortices are expected to be co-rotating with the crust, including the proton condensate and the lattice of fluxoids, in the core of the star. Strict co-rotation of the vortices with the crust is not however possible for an assumed vortex relaxation process due only to the electron scattering. In contrast, the pinning force could be imparted even while the vortices are co-rotating with the fluxoids. The pinning potential barrier could act as a "stretched spring", that does pull the mass attached to it, even while both are moving together. In any case, the difference in the rotation rate between the vortices and the fluxoids (the crust) is tiny and will be neglected, for the steady state considered. On the other hand, the steady-state rotation rate of the superfluid should be different than that of the crust and/or the vortices, due to the assumed pinning of the vortices. The superfluid rotational lag is defined as $\omega=\Omega_{\mathrm{S}}-\Omega_{\mathrm{L}}$, where $\Omega_{\mathrm{L}}$ is the rotation frequency of the neutron vortex lines. 


\subsubsection{The Steady-state Lag}

The superfluid critical lag $\omega_{\text {cr }}$ is determined from a balance of the radial Magnus force $F_{\mathrm{Mr}}=\rho_{\mathrm{s}} \kappa r \omega$ with the radial component of the external force, $F_{\mathrm{r}}$, on the vortices, per unit length. Equating $F_{\mathrm{r}}$ with the pinning force results in (Ding et al. 1993)

$$
\begin{aligned}
\omega_{\mathrm{cr}} & =\frac{f_{\mathrm{P}}}{\rho_{\mathrm{s}} \kappa r d_{\mathrm{f}}} \\
& \sim 1.6 \times 10^{-4}\left(\mathrm{rad} \mathrm{s}^{-1}\right) \frac{B_{12}^{1 / 2}}{r_{6}}
\end{aligned}
$$

where $r_{6}$ is the value of $r$ in units of $10^{6} \mathrm{~cm}$, and $B_{12}$ is the strength of the magnetic field $B_{\mathrm{c}}$ in the stellar core in units of $10^{12} \mathrm{G}$. For the typical magnetic fields of young pulsars, one finds typical values of

i) $\omega_{\mathrm{cr}} \gtrsim 10^{-4} \mathrm{rad} \mathrm{s}^{-1}$,

for $R_{6} \sim 1$, at the outer regions of the stellar core, and

ii) $\omega_{\mathrm{cr}} \gtrsim 10^{-3} \mathrm{rad} \mathrm{s}^{-1}$,

for $R_{6} \sim 0.1$, at the inner parts, embracing $\sim 1 \%$ of the moment of inertia of the core.

The steady-state spinning down will be thus established while $\Omega_{\mathrm{c}}=\Omega_{\mathrm{L}}<\Omega_{\mathrm{s}}, \dot{\Omega}_{\mathrm{s}}=\dot{\Omega}_{\mathrm{c}}$, and the steady-state vale of the lag $\omega_{\infty}=\omega_{\text {cr }}>0$, with the above estimates for its value (which also indicates a state of differential rotation for the core superfluid at the different radial distances). It may be noted that random unpinning of the vortices could in principle serve to establish even smaller values of the lag $\omega_{\infty}<\omega_{\mathrm{cr}}$. An estimate of the effect, based

on the vortex creep model, shows however the difference to be negligible (Chau et al. 1992). Thus, we will be neglecting the role of random unpinning of the vortices, and hence assuming $\omega_{\infty}=\omega_{\mathrm{cr}}$, in the following.

The expected relative rotation of the "crust", the core superfluid, and its vortices is sketched in Fig. 1, as a visual aid which turns out to be vital too. The sketch helps to keep track and be ensured that the, preliminary but, fundamental physics applicable to a transfer of angular momentum between the superfluid and its environment (the crust, here) is obeyed! That is, for a superfluid spin-down (-up) to be achieved the crust must be, or tend to be, rotating slower (faster) than not only the superfluid itself, but also slower (faster) than the vortices (see $\S 3.2 .2$ and $\S 4$, below). Each plot indicates the three successive stages at a disturbance and the transitions between them. Namely, the conditions during a steady state, as discussed above (marked as "S.S."), followed by a jump in the rotation frequency of the crust ("JUMP"), and after the core has responded to that jump, returning to a new 
steady state ("post-G"). The cases of pinned and free vortices behave differently and are shown separately, as there is also a further distinction for the pinned cases depending on the relative size of the jump, to be discussed below.

\subsection{Vortex Velocity Relaxation}

An assumed departure from the steady-state co-rotation of the vortices with the fluxoids brings about their continually crossing one another, with an energy cost of the pinning energy $E_{\mathrm{P}}$, per intersection, associated to a frictional force $f_{\mathrm{P}}$. The total number $N_{\mathrm{X}}$ of crossings per relative rotation cycle of the two lattices (embedded in a spherical boundary surface) is $N_{\mathrm{X}}=N_{\mathrm{f}} N_{\mathrm{v}} \sin \chi$ (see the Appendix), where $N_{\mathrm{f}}$ and $N_{\mathrm{v}}$ are the total number of the fluxoids and the vortices, and $\chi$ is the angle between the two families of lines, namely the angle of inclination between the rotation and the magnetic axes of the star. Excluding the cases of parallel lattices corresponding to $\chi \sim 0$ or $180 \mathrm{deg}$ which are not common among the observed pulsars, the correction due to the $\sin \chi$ factor may be neglected, assuming near perpendicular geometry between the two lattices, for simplicity.

The effective frictional force $F_{\text {pin }}$ of the pinning, per unit length of a vortex, while cutting through the lattice of uniformly distributed fluxoids (with a spacing $d_{\mathrm{f}}$ ) may be estimated by taking a time average over a cycle ("pinning cycle") of passing through a pinning region, of a length $l_{1}=d_{P}$, and an adjacent inter-pinning zone, of a length $l_{2}=d_{\mathrm{f}}-d_{P}$. This is in contrast to the drag force due to the electron scattering $F_{\text {elec }}$, which acts continuously, and will be treated separately. Thus,

$$
F_{\text {pin }}=\frac{1}{T_{\text {cyc }}}\left(t_{1} f_{1}+t_{2} f_{2}\right)
$$

where $T_{\text {cyc }}=t_{1}+t_{2}$ is the total time for a vortex to pass through one pinning cycle, and the subscripts refer to the two regions, respectively. The pinning force is operative only within each pinning zone, namely

$$
\begin{aligned}
f_{1} & =N_{\mathrm{c}} f_{\mathrm{P}} \\
& =\frac{f_{\mathrm{P}}}{d_{\mathrm{f}}}=\frac{E_{\mathrm{P}}}{d_{\mathrm{P}} d_{\mathrm{f}}}, \\
f_{2} & =0,
\end{aligned}
$$

where we have used $N_{\mathrm{c}}=\frac{1}{d_{\mathrm{f}}}$ for the number $N_{\mathrm{c}}$ of the potential crossings per unit length of a vortex, per pinning cycle (see the Appendix).

Next, the microscopic motion of any given vortex, while cutting through the fluxoids, 
might be expected to fall in between the following two limiting types of behaviors, for a first approximation.

1. A vortex might preserve the same (relative) velocity (with the fluxoids) in both the above two regions, throughout a pinning cycle. Hence, the travel times, for the two regions, would be linearly proportional to their sizes. Namely,

$$
\begin{aligned}
\frac{t_{1}}{t_{2}} & =\frac{l_{1}}{l_{2}}=\frac{d_{P}}{d_{\mathrm{f}}-d_{P}}, \text { and } \\
\frac{t_{1}}{T_{\mathrm{cyc}}} & =\frac{d_{P}}{d_{\mathrm{f}}}
\end{aligned}
$$

which results in

$$
\begin{aligned}
F_{\text {pin }} & =\frac{E_{\mathrm{P}}}{d_{\mathrm{f}}^{2}} \\
& \sim 6 \times 10^{12}\left(\text { dyn } \mathrm{cm}^{-1}\right) E_{\mathrm{MeV}} B_{12}
\end{aligned}
$$

where $E_{\mathrm{MeV}}$ is the value of $E_{\mathrm{P}}$ in units of $\mathrm{MeV}$.

2. Or, the vortex might be expected to adjust its velocity instantaneously as it moves through the pinning and the free regions, successively. The vortices might therefore spend almost all of the time inside the pinning regions and fly across the free spacings between the fluxoids rapidly, in almost zero time. That is

$$
\begin{aligned}
& t_{2}=0, \text { and } \\
& t_{1}=T_{\text {cyc }}
\end{aligned}
$$

which results in

$$
\begin{aligned}
F_{\text {pin }} & =\frac{E_{\mathrm{P}}}{d_{\mathrm{f}} d_{\mathrm{P}}} \\
& \sim 1.4 \times 10^{16}\left(\text { dyn } \mathrm{cm}^{-1}\right) E_{\mathrm{MeV}} d_{12}^{-1}
\end{aligned}
$$

where $d_{12}$ is the value of $d_{\mathrm{P}}$ in units of $10^{-12} \mathrm{~cm}$.

The above two cases might each represent a better approximation for either a transient relaxation, following a sudden change of the rotation frequency of the fluxoids (as for a glitch) or during an equilibrium steady state spin-down phase, respectively. In either case, the force is independent of the relative velocity of the two families of lines; a dynamical feature different than that of, say, the force due to the electron scattering. 
The corresponding vortex relaxation time $T_{\mathrm{P}}$, needed for a given relative velocity between the fluxoids and vortices to be dissipated, may be defined, from the EOM of a unit volume of the charged component gas co-rotating with the fluxoids (the "crust"), through

$$
n_{\mathrm{v}} \vec{F}_{\text {pin }}=\rho_{\mathrm{c}} \frac{\overrightarrow{v_{\mathrm{L}}}-\overrightarrow{v_{\mathrm{c}}}}{T_{\mathrm{P}}}
$$

where $\overrightarrow{v_{\mathrm{c}}}$ is the local velocity of the electron gas and the fluxoids, ie. the "crust", and $\rho_{\mathrm{c}}$ is its effective density, corresponding to the contribution of the real crust together with the free protons (and the electrons) in the moment of inertia of the star. Note that since $F_{\text {pin }}$ is a velocity-independent force, $T_{\mathrm{P}}$ is the total time for the decay of the velocity, and not an exponential time constant. For an assumed jump $\Delta \Omega_{\mathrm{c}}$ in the rotation frequency $\Omega_{\mathrm{c}}$ of the crust, out of its steady state co-rotation with the vortices, one derives, from Eq. 16 together with Eq. 11 or 15,

$$
\begin{aligned}
T_{\mathrm{P}} & =830(\mathrm{~s}) \frac{\Delta \Omega_{\mathrm{c}}}{\Omega_{\mathrm{c}}} \frac{\rho_{13}}{E_{\mathrm{MeV}} B_{12}}, \text { or } \\
& =0.4(\mathrm{~s}) \frac{\Delta \Omega_{\mathrm{c}}}{\Omega_{\mathrm{c}}} \frac{\rho_{13} d_{12}}{E_{\mathrm{MeV}}},
\end{aligned}
$$

for the two approximations considered above, respectively, and where $\rho_{13}$ is the value of $\rho_{\mathrm{c}}$ in units of $10^{13} \mathrm{~g} \mathrm{~cm}^{-3}$. Notice that the dependence on the distance $r$ from the rotation axis $(v=r \Omega)$ has been averaged out, and the radial velocity of the vortices, during a relaxation, would have negligible effect.

Substituting typical values of $\frac{\Delta \Omega_{\mathrm{c}}}{\Omega_{\mathrm{c}}} \sim 10^{-6}$, and $E_{\mathrm{MeV}} \sim \rho_{13} \sim B_{12} \sim 1$, one finds, from Eq. 17, $T_{\mathrm{P}} \sim 10^{-4} \mathrm{~s}$, which is much shorter than the corresponding time scale due to the electron scattering $\tau_{\mathrm{e}} \sim 1-2 \mathrm{~s}$ (Alpar et al. 1984b). The estimated value of $T_{\mathrm{P}}$ for the other case, from Eq. 18, is even smaller, by more than three orders of magnitudes. Nevertheless, because $T_{\mathrm{P}}$ does depend on the initial value of the induced relative velocity it may, in principle, become larger than $\tau_{\mathrm{e}}$, but that might happen only for the very large assumed disturbances, much larger than that observed even in the giant glitches of the Vela pulsar. For the steady state spin down, on the other hand, $T_{\mathrm{P}}$ would be even much smaller than the above estimate.

The drag force, $\vec{F}_{\text {elec }}$, of the electron scattering off neutron vortices is, likewise, associated with a vortex relaxation time $\tau_{\mathrm{e}}$, through an equation similar to Eq. 16, as is the relation between the overall relaxation time scale $\tau_{v}$ with the total force $\vec{F}_{\text {tot }}=\vec{F}_{\text {pin }}+\vec{F}_{\text {elec }}$, namely

$$
n_{\mathrm{v}} \vec{F}_{\text {tot }}=\rho_{\mathrm{c}} \frac{\overrightarrow{\mathrm{L}}-\overrightarrow{v_{\mathrm{c}}}}{\tau_{v}}
$$

which means

$$
\frac{1}{\tau_{v}}=\frac{1}{T_{\mathrm{P}}}+\frac{1}{\tau_{\mathrm{e}}}
$$


Therefore, the frictional pinning force turns out to be the dominant coupling mechanism for the core superfluid, and $\tau_{v} \sim T_{\mathrm{P}}$ may be adopted, in general.

\section{Coupling Timescale of the Superfluid}

The dynamical timescale for the rotational coupling of the bulk superfluid to its environment (the crust) is the time needed for the simultaneous re-adjustment of the vortices in both radial and azimuthal directions in response to an external torque on the superfluid, exerted primarily on the vortices. The external forces on the vortices could, in general, be of a viscous drag or a "static" frictional nature (Adams et al. 1985; Jones 1991b). For the superfluid in the core of a neutron star, both types are present and correspond to the electron scattering and the fluxoid "scattering", discussed above, respectively. The latter type, associated with the "pinning" forces, should not be however confused with the role of the pinning forces on the pinned vortices co-rotating with the pinning centers. In order for the pinning forces to act as frictional forces and impart a net torque on the superfluid the vortices should maintain a radial velocity, hence unpinning continuously due to the effect

of the Magnus force, which requires $|\omega| \geq \omega_{\text {cr }}$ (Adams et al. 1985; Jahan-Miri 2005a). For $|\omega|<\omega_{\text {cr }}$, random unpinning events might play a role in the long-term coupling of the superfluid, but the effect is neglected here, for simplicity and more so because of its negligible effects on the quick post-glitch responses. A more general treatment should include the superfluid coupling rate driven also by the random unpinning of the vortices (Jahan-Miri 2006), as has been already studied, in the context of the vortex creep model, and found to be unimportant (Sidery \& Alpar 2009).

\subsection{The case of free vortices (no pinning barriers)}

The dynamical timescale $\tau_{\text {free }}$ of the superfluid rotational relaxation, in the absence of any pinning of the vortices (Fig. 1a), has been previously determined (Alpar \& Sauls 1988; Jahan-Miri 1998) as

$$
\tau_{\text {free }}=\frac{I_{\mathrm{s}}}{I} \tau_{\mathrm{e}}+\frac{I_{\mathrm{c}}^{2} P_{\mathrm{s}}^{2}}{16 \pi^{2} I_{\mathrm{s}} I} \tau_{\mathrm{e}}^{-1}
$$

This is the exponential timescale that appears in the solutions obtained for the relaxations of the radial $r_{\mathrm{v}}(t)$ and the azimuthal $\phi_{\mathrm{v}}(t)$ components of the vortex positions, in polar coordinates on the equatorial plane, as a function of time $t$. The time behavior of the vortex position (following an assumed sudden rise in the rotation frequency of the "crust", say at a 
glitch) is governed by the vortex equation of motion (Eq. 1), using $\vec{F}_{\text {ex }}=\vec{F}_{\text {elec }}$, in the absence of any pinning. The solutions, which were not given in the correct form previously, are:

$$
\begin{aligned}
& r_{\mathrm{v}}(t)=r_{0}\left[\frac{\Omega_{\mathrm{s} 0}}{\Omega_{\mathrm{F}}}+\left(1-\frac{\Omega_{\mathrm{s} 0}}{\Omega_{\mathrm{F}}}\right) e^{-t / \tau_{\text {free }}}\right]^{1 / 2} \\
& \phi_{\mathrm{v}}(t)=\phi_{0}+\Omega_{\mathrm{F}} t+\left(\frac{I_{\mathrm{s}} \kappa n_{\mathrm{v}}}{I} \tau_{e}-\frac{I_{\mathrm{c}}}{I \kappa n_{\mathrm{v}}} \tau_{e}^{-1}\right) \ln \left(\frac{r_{\mathrm{v}}(t)}{r_{0}}\right)
\end{aligned}
$$

where 0 -subscripts indicate initial values at $t=0$ of the corresponding quantities, $I=I_{\mathrm{c}}+I_{\mathrm{s}}$, and $\Omega_{\mathrm{F}}=\frac{1}{I}\left(I_{\mathrm{c}} \Omega_{\mathrm{c} 0}-I_{\mathrm{s}} \Omega_{\mathrm{s} 0}\right)$ is the final equilibrium frequency of both the crust and the superfluid.

\subsection{The case of Pinned Superfluid}

For a pinned superfluid, already in a steady-state with $\Omega_{\mathrm{c}}=\Omega_{\mathrm{L}}<\Omega_{\mathrm{s}}$ and $\omega=\omega_{\infty}=$ $\omega_{\text {cr }}>0$, as discussed in $\S 2.1$, a sudden jump in $\Omega_{\mathrm{c}}$ is then assumed to occur, the cause of which is irrelevant for the purpose of the present discussion. The crossing through the fluxoids by the vortices is inevitable until the state of co-rotation between them is reached (considering rigid vortex lines), hence the vortices would be subject to forward azimuthal pinning forces. The response of the pinned superfluid to such disturbances is argued, below, to be qualitatively different, depending on the magnitude of the increase $\Delta \Omega_{\mathrm{c}}$ in the rotation frequency of the crust. The effect, should not be however attributed to the particular form(s) of the pinning force, calculated above. Indeed, the same types of behaviors would be expected even in the presence of the drag force due to the electron scattering alone, as long as the neutron vortices are assumed to be subject to the pinning to the "crust" (co-rotating with the fluxoids and the charged components). Also, note that the decomposition of the effect into a "jump" in $\Omega_{\mathrm{c}}$ and a "subsequent response" of the superfluid is not meant to be in real time, rather it is for the sake of the analysis, as is common.

\subsubsection{Large Jump}

Following an assumed "large" jump, such that $\Delta \Omega_{\mathrm{c} 0}>\omega_{\mathrm{cr}}$ (Fig. 1c), the fluxoids (and the electron gas) would be rotating faster than both the vortices and the superfluid itself. Hence, the existing forward azimuthal forces on the vortices would be consistent with a corresponding inward motion of the vortices required for a spin-up torque to be imparted to

the superfluid. The steady state lag would be thus washed out during (the jump and) the following quick relaxation of the superfluid (and the vortices), bringing the superfluid, first, 
to a state of co-rotation with the fluxoids and the vortices. Hence, following a large jump the superfluid will be, first, spun up by the crust, on a dynamical time scale $\tau_{\text {pin }}$, until the equilibrium state $\Omega_{\mathrm{s}}=\Omega_{\mathrm{L}}=\Omega_{\mathrm{c}}$ is reached (see Fig. 1c).

The coupling timescale $\tau_{\text {pin }}$ may be determined from a calculation similar to the above case of the free vortices, except that $\tau_{v}$ (Eq. 20) would now be the relevant vortex relaxation time. That is (compare with Eq. 21)

$$
\tau_{\text {pin }}=\frac{I_{\mathrm{s}}}{I} \tau_{v}+\frac{I_{\mathrm{c}}^{2} P_{\mathrm{s}}^{2}}{16 \pi^{2} I_{\mathrm{s}} I} \tau_{v}{ }^{-1} .
$$

It may be noted that, the pinning force, $f_{\mathrm{P}}$, at each intersecting point, is directed solely along the direction perpendicular to the intersecting fluxoid; the component of the force along

the fluxoid being zero (Sauls 1989; Sidery \& Alpar 2009). In solving the vortex equation of motion (Eq. 1), with the substitution $\vec{F}_{\text {ex }}=\vec{F}_{\text {pin }}$, a decomposition of the force to the radial and azimuthal components would be initially faced with a difficulty, that may be however bypassed. The projections of the fluxoids, on the equatorial plane, form an array of parallel lines. In the presence of a difference between the rotation rates of the vortices and the fluxoids, the vortices at any given radial distance $r$ cut through those lines at varying angles. Hence, the azimuthal component of the pinning force, at each intersection, would vary between its maximum vale $f_{\mathrm{P}}$ down to zero, for the vortices located at the different azimuth angles. The same would be true for the radial component of the force, as well. Thus, at any given time and for the vortices at any given radial distance, the average values of the azimuthal and the radial components of the pinning force, per intersection, may be used; averaged over the vortices at varying azimuthal angles over a circle. The average values, would be the same for both components and, simply amount to $\frac{2}{\pi} f_{\mathrm{P}}$. This will correspond to a change in the relaxation time $T_{\mathrm{P}}$ by a factor $\frac{\pi}{2}$, that we have neglected.

\subsubsection{Small Jump}

In contrast, following an assumed "small" jump, in the rotation rate of the crust, such that $\Delta \Omega_{\mathrm{c} 0}<\omega_{\mathrm{cr}}$ (Fig. 1b), there would follow no transfer of angular momentum between the crust and the superfluid, initiated by the jump. The superfluid could be neither spun down nor up, by the crust! Following the jump, the vortices would be indeed spun up to come into a co-rotation with the fluxoids, due to the existing forces. Nevertheless, the superfluid which is already rotating faster than the crust, and its own vortices, (Fig. 1b) could not be possibly spun up, and gain angular momentum from the slower component, during such a spin-up of the vortices. Also, the superfluid could not be spun down, during such a spinning up of the crust, that is accompanied by a spinning up of the vortices. Because, a spin-down 
of the superfluid requires a radially outward motion of the vortices. Nonetheless, an outward motion of the vortices may be realized only if they do rotate, or tend to be rotating, faster than the the superfluid environment, so that a slowing down torque could be imparted on them (see Jahan-Miri (2005a, b) for a more extended explanation; indeed false conclusions, about the behavior of the superfluid in the crust of a neutron star, have been communicated because of the neglect of this primitive but fundamental fact, as discussed therein).

Hence, following a small jump, the co-rotation of the vortices with the crust is achieved with no transfer of angular momentum to the superfluid, ie. at constant $\Omega_{\mathrm{s}}$. The time scale involved would be only that of the azimuthal vortex velocity relaxation, $T_{\mathrm{P}}$. The speeding up of the vortices alone does not, however, require any transfer of angular momentum, since these are but massless (super)fluid configurations (neglecting the inertia of the vortices, as is usually assumed).

\section{Discussion and Observational Implications}

Glitches are observed in radio pulsars as sudden changes $\Delta \Omega_{\mathrm{c}}$ in the rotation frequency $\Omega_{\mathrm{c}}$ of the crust with observed values of the jump in the range $10^{-9} \lesssim \frac{\Delta \Omega_{\mathrm{c}}}{\Omega_{\mathrm{c}}} \lesssim 10^{-6}$. In younger pulsars, the jump in $\Omega_{\mathrm{c}}$ is also accompanied by an increase $\Delta \dot{\Omega}_{\mathrm{c}}$ in the observed spin-down rate $\dot{\Omega}_{c}$ of the crust, with typical values of $\frac{\Delta \dot{\Omega}_{\mathrm{c}}}{\dot{\Omega}_{\mathrm{c}}} \lesssim 2.5 \%$ (Lyne 1987; Krawczyk et al. 2003). The effect, as such, has been explained successfully in terms of a decoupling of the superfluid in the crust of a neutron star, which has a fractional moment of inertia of similar magnitudes (Alpar et al. 1984a). However, there has been observed cases with

$$
\frac{\Delta \dot{\Omega}_{\mathrm{c}}}{\dot{\Omega}_{\mathrm{c}}}>10 \%
$$

and recovery timescales up to $\sim 44 \mathrm{~d}$ (Lyne 1987; Flanagan 1995). These observed cases would necessarily imply that part of the star with the same fractional moment of inertia( ie. $>10 \%$, and indeed up to $60 \%$ at some observations) has been decoupled from the crust, at the time of the observation. This, by itself, is a definite proof of a rotational decoupling of (a part of) the core of the star, at those glitches, since the crust does not constitute that much of the moment of inertia of the star. Given also the observed recovery time scales at these events, the effect, in turn, indicates that a pinned superfluid component of the stellar core must be involved, as has been argued previously (Jahan-Miri 2005b). The core superfluid, in the absence of pinning, does similarly cause observed large values of $\frac{\Delta \dot{\Omega}_{\mathrm{c}}}{\dot{\Omega}_{\mathrm{c}}}$, but that would last only over a time of the order of $\tau_{\text {free }}$ (Eq. 21). In contrast, the pinned superfluid remains decoupled until the superfluid rotational lag, which is washed out quickly at the jump (see Figs $1 \mathrm{~b} \& 1 \mathrm{c})$, recovers its steady state value $\omega_{\mathrm{cr}}$. During this recovery period $t_{\mathrm{PG}}$, the 
observable crust is being spun down, by the unchanged external torque $N_{\text {ext }}$, at an increased post-glitch rate $\dot{\Omega}_{\mathrm{PG}}$, that could be as large as,

$$
\dot{\Omega}_{\mathrm{PG}}=\frac{I}{I_{\mathrm{c}}} \dot{\Omega}_{\mathrm{SS}}
$$

where $\dot{\Omega}_{\mathrm{SS}}=\frac{N_{\text {ext }}}{I}$ is the steady state value of the spin down rate of the star. Since, the vortices are pinned and co-rotating with the fluxoids and the crust, thus the superfluid is decoupled and the lag is built up due only to the resulting decrease in $\Omega_{\mathrm{c}}$. Hence, the post-glitch recovery timescale $t_{\mathrm{PG}}$, over which the increased spin-down rate of the crust may persist, is expected to be

$$
t_{\mathrm{PG}}=\frac{\omega_{\mathrm{cr}}}{\dot{\Omega}_{\mathrm{PG}}} \gtrsim 10^{5}(\mathrm{~s})
$$

where the estimated value is for an assumed low value of $\omega_{\text {cr }}=10^{-4} \mathrm{rad} \mathrm{s}^{-1}$, a large value of $\frac{I}{I_{\mathrm{c}}}=20$, and a typical value of $\dot{\Omega}_{\mathrm{c}}=10^{-10} \mathrm{rad} \mathrm{s}^{-2}$. Obviously, larger recovery times are expected for the lower values of $\dot{\Omega}_{\mathrm{PG}}$, that would be expected if only a fraction of the core superfluid takes part in the decoupling-coupling mechanism. Therefore, the largest observed values for the post-glitch spin-down rates of the pulsars, as well as the associated recovery timescales recorded, find a natural explanation in terms of the expected decoupling of the superfluid component in the core, being subject to the pinning with the fluxoids.

Further, the two cases of the large versus the small jumps, considered above, would have different implications for the observable post-glitch recovery. In the case of a large jump, the expected initial spin-up of the core superfluid, discussed above, would show up as a further increase, by an additional factor $\frac{I}{I_{\mathrm{c}}}$, in the observable spin-down rate of the crust, compared to that given in Eq. 26. In contrast, no such an increase is expected for the small jumps (compare Fig. 1b with 1c). Such a difference in behavior, between the two cases, would however persist only over time periods $\sim \tau_{\text {pin }}$, which makes it indeed hard to be detected, observationally.

On the other hand, the two cases might be judged and distinguished based also on the earliest detected values of $\Delta \Omega_{\mathrm{c}}$, as compared to the value of $\omega_{\mathrm{cr}}$. That is, the two cases of small versus large jumps correspond to (initial) values of $\Delta \Omega_{\mathrm{c}}<\omega_{\mathrm{cr}}$ and $\Delta \Omega_{\mathrm{c}}>\omega_{\mathrm{cr}}$, respectively (as indicated in Figs $1 \mathrm{~b}$ and 1c). Notice that, for a large jump the condition $\Delta \Omega_{\mathrm{c}}>\omega_{\text {cr }}$ would be still true, even after the initial relaxation of the superfluid, over the time $\tau_{\text {pin }}$, ie. at the post-glitch $t=0$ on Fig. 1c. Also, the observational uncertainty in the exact epoch of the glitch, being of the order of few minutes, would not blur between the two cases, since the change in $\Omega_{\mathrm{c}}$ over such periods would be negligible. Therefore, given the generally observed initial values for $\frac{\Delta \Omega_{\mathrm{c}}}{\Omega_{\mathrm{c}}}$, cited above, as compared to the expected values of 
$\omega_{\text {cr }} \gtrsim 10^{-4} \mathrm{rad} \mathrm{s}^{-1}$ for the pinned core superfluid, one may conclude that the case of a small jump is the relevant one for most, if not all, of the observed glitches.

Moreover, the two cases would have different implications also for the possible cause of the glitches. A large jump has to be necessarily induced by some "external" agent, other than the core superfluid itself, simply because a donor of the angular momentum could not spin up its counterpart to a frequency more than its own. In contrast, and by the same token, a glitch induced by the core superfluid would necessarily rank as a small one. The core superfluid is thus, statistically, more favored as the potential cause of the small jumps. The above mentioned indication of the data for the prevalence of the small jumps, would thus further support the glitch inducing mechanisms driven by the core superfluid (Muslimov \& Tsygan 1985; Jahan-Miri 2002).

Finally, the pinned core superfluid does not respond to a jump in $\Omega_{\mathrm{c}}$, at a glitch, smaller than the critical value of the superfluid rotational lag, $\omega_{\mathrm{cr}}$, as indicated above. The angular momentum gained by the crust, at such glitches, would not be shared, any further, with the core superfluid. The case is highlighted here again, not because of any prominent observable effect different than the other case of the large jumps. Rather, for its prominent conceptual value in demonstrating the vital requirement for the presence of a corresponding torque for a spin-down (-up) of a superfluid to be achieved. The motion of the superfluid vortices too has to conform with this basic physics, be it a smooth motion in the absence of any pinning, or the so-called creeping of the pinned vortices.

This work was supported by a grant from the Research Committee of Shiraz University.

\section{A. The average rate of crossing with fluxoids, per unit length of a vortex}

We are assuming the fluxoids, in the core of a neutron star, form a uniform array of parallel lines, along the magnetic axis $(\vec{B})$ of the star, having a total number of $N_{\mathrm{f}}$, within a spherical boundary surface of radius $R$. Likewise, the neutron vortices constitute a uniform lattice of parallel lines, along the rotation axis $(\vec{\Omega})$ of the star, having a total number of $N_{\mathrm{v}}$, within the same spherical boundary. The two axes are in general inclined at an angle $\chi$, of which we consider first the case with $\chi=\frac{\pi}{2}$, for further clarity, and will generalize at the end. Taking the z-axis along $\vec{\Omega}$, the fluxoids are thus parallel to the $\mathrm{x}-\mathrm{y}$ plane.

1. A circular slab, perpendicular to the z-axis, its rim at the polar angle $\theta$, a distance $z=R \cos \theta$ from the origin, with a radius $R_{\mathrm{z}}=R \sin \theta$, and a thickness $d z=\sin \theta d \theta$, 
includes a number $N_{\mathrm{b}}$ of fluxoids, being parallel to it, where

$$
\begin{aligned}
N_{\mathrm{b}} & =\frac{N_{\mathrm{f}}}{\pi R^{2}}\left(2 R_{\mathrm{z}} d z\right) \\
& =\frac{2}{\pi} N_{\mathrm{f}} \sin ^{2} \theta d \theta
\end{aligned}
$$

2. The fraction of the fluxoids in the slab, lying at (cylindrical) distances $r$ to $r+d r$ from the $\mathrm{z}$-axis is

$$
N_{\mathrm{s}}(r)=\frac{d r}{2 R_{\mathrm{z}}} N_{\mathrm{b}}
$$

3. Each of the lines at $r$, sweeps an annular area $s(r)$, in the $\mathrm{x}$-y plane, per rotation cycle, that is

$$
s(r)=2\left(\pi R_{\mathrm{z}}^{2}-\pi r^{2}\right)
$$

4. The area $d S_{\mathrm{b}}$ swept by all the fluxoids in the slab would be

$$
\begin{aligned}
d S_{\mathrm{b}} & =2 \int_{0}^{R_{\mathrm{z}}} N_{\mathrm{s}}(r) s(r) \\
& =\frac{4}{3} \pi R_{\mathrm{z}}^{2} N_{\mathrm{b}}
\end{aligned}
$$

5. The cumulative total area $S_{\text {tot }}$ swept by all the fluxoids within the sphere, parallel to the $\mathrm{x}-\mathrm{y}$ plane, thus becomes

$$
\begin{aligned}
S_{\mathrm{tot}} & =2 \int_{\theta=0}^{\frac{\pi}{2}} d S_{\mathrm{b}} \\
& =\pi R^{2} N_{\mathrm{f}}
\end{aligned}
$$

This may be compared with a corresponding value $\frac{4}{3} \pi R^{2} N_{\mathrm{f}}$ which would result for an assumed cylindrical geometry, or with a value $2 \pi R^{2} N_{\mathrm{f}}$ if all the fluxoids were of the same length $2 R$, lying radially.

6. The number $N_{\mathrm{X}}$ of crossings between the fluxoids and the vortices (the latter all passing through the x-y plane, with a number density $n_{\mathrm{v}}=\frac{N_{\mathrm{v}}}{\pi R^{2}}$ ), per cycle of relative rotation, per unit area of the $\mathrm{x}-\mathrm{y}$ plane, becomes

$$
N_{\mathrm{X}}=n_{\mathrm{v}} S_{\text {tot }}=N_{\mathrm{v}} N_{\mathrm{f}}
$$

which may be, in turn, compared with a corresponding value $2 N_{\mathrm{v}} N_{\mathrm{f}}$, if each fluxoid were to cross each and any of the vortices, per relative rotation cycle. 
7. The total sum $L_{\mathrm{v}}$ of the lengths of all the vortices, within the sphere, is

$$
L_{\mathrm{v}}=n_{\mathrm{v}}\left(\frac{4}{3} \pi R^{3}\right)=\frac{4}{3} R N_{\mathrm{v}}
$$

Hence, the average number $N_{1}$ of crossings, per unit length of a vortex, per rotation cycle, is

$$
N_{1}=\frac{N_{\mathrm{X}}}{L_{\mathrm{v}}}=\frac{3}{4} \frac{N_{\mathrm{f}}}{R}
$$

8. For an square lattice of the fluxoids, with a spacing $d_{\mathrm{f}}$, between the nearest fluxoids,

$$
N_{\mathrm{f}}=\frac{\pi R^{2}}{d_{\mathrm{f}}^{2}}
$$

Thus,

$$
N_{1}=\frac{3}{4} \frac{\pi R}{d_{\mathrm{f}}^{2}}
$$

9. The point of intersection of each vortex with the $\mathrm{x}-\mathrm{y}$ plane, at a distance $r$ from the z-axis, moves a distance $d(r)=2 \pi r$, per rotation cycle. The corresponding average distance $d_{\mathrm{v}}$ that a vortex moves, becomes

$$
d_{\mathrm{v}}=\frac{1}{R} \int_{0}^{R} d(r) d r=\pi R
$$

10. Therefore, the rate $N_{\mathrm{c}}$ of crossings, per unit length of a vortex, per pinning cycle, is derived as

$$
N_{\mathrm{c}}=\frac{d_{\mathrm{f}}}{d_{\mathrm{v}}} N_{1}=\frac{3}{4} \frac{1}{d_{\mathrm{f}}}
$$

11. For the general case of an arbitrary inclination angle $\chi$, the derivation would be similar, except that one starts by considering a slab, parallel to the fluxoids and at the same angle $\chi$ with respect to the rotation axis, so that it preserves its angle during the rotation. Then the projected area swept by those fluxoids, on the $\mathrm{x}-\mathrm{y}$ plane would have an additional factor $\sin \chi$, as compared to that in Eq. A8. As a consequence, the total number of the crossings would be corrected by the same factor, namely $N_{\mathrm{X}}=N_{\mathrm{v}} N_{\mathrm{f}} \sin \chi$, and also for the final result, $N_{\mathrm{c}}=\frac{3}{4} \frac{1}{d_{\mathrm{f}}} \sin \chi$. 


\section{REFERENCES}

Adams P. W., Cieplak M., Glaberson W. I. 1985, Phys. Rev. B, 32, 171

Alpar M. A., Anderson P. W., Pines D., Shaham J. 1984a, ApJ, 276, 325

Alpar M. A., Langer S. A., Sauls J. A. 1984b, ApJ, 282, 533

Alpar M. A., Sauls J. A. 1988, ApJ, 327, 723

Chau H. F., Cheng K. S., Ding K. Y. 1992, ApJ, 399, 213

Ding K. Y., Cheng K. S., Chau H. F. 1993, ApJ, 408, 167

Flanagan C. S., in Alpar M. A., Kiziloğlu Ü., van Paradijs J., eds, Proc. NATO ASI C450, The Lives of the Neutron Stars. Kluwer, Dordrecht, p. 181 (1995)

Jahan-Miri M., Bhattacharya D. 1994, MNRAS, 269, 455

Jahan-Miri M. 1996a, MNRAS, 283, 1214

Jahan-Miri M. 1998, ApJ, 501, L185

Jahan-Miri M. 2000, ApJ, 532, 514

Jahan-Miri M. 2002, MNRAS, 330, 279

Jahan-Miri M. 2005a, J. Low Temp. Phys., 139, 371

Jahan-Miri M. 2005b, New Astr., 11, 157

Jahan-Miri M. 2006, ApJ, 650, 326

Jones P. B. 1991a, MNRAS, 253, 279

Jones P. B. 1991b, ApJ, 373, 208

Jones P. B. 2006, MNRAS, 365, 339

Krawczyk A., Lyne A. G., Gil J. A., Joshi B. C. 2003, MNRAS, 340, 1087

Link B. 2009, Phys. Rev. Lett., 102, 131101

Lyne A. G. , Nature, 326, 569 (1987)

Muslimov A. G., Tsygan A. I. 1985, Ap\&SS, 115, 43 
Sauls J. A. 1989, in Ögelman H., van den Heuvel E. P. J., eds, Proc. NATO ASI 262, Timing Neutron Stars. Kluwer, Dordrecht, p.

Sedrakian A. D., Sedrakian D. M. 1992, JETP, 75, 395

Sidery T., Alpar M. A. 2009, MNRAS, 400, 1859

Sonin E. B. 1987, Rev. Mod. Phys., 59, 87

Srinivasan G., Bhattacharya D., Muslimov A. G., Tsygan A. I. 1990, Curr. Sci., 59, 31

Tsakadze J. S., Tsakadze S. J. 1980, J. Low Temp. Phys., 39, 649

Urpin V., Geppert U., Konenkov D. 1997, MNRAS, 295, 907 
Fig. 1.- Schematic representation of the relative values of the angular velocities of the superfluid $\Omega_{\mathrm{s}}$ (dotted line), the vortices $\Omega_{\mathrm{L}}$ (dashed line), and the "crust" $\Omega_{\mathrm{c}}$ (full line), excluding the overall spinning down of the system. On each plot, three successive phases are shown at a relaxation, namely an earlier steady state (marked as "S.S."), an assumed sudden increase in $\Omega_{\mathrm{c}}$ ("JUMP") and the subsequent relaxed state ("POST-G"). a) is for the case of free vortices, in the absence of pinning barriers, whence the steady state spinning down corresponds to (an almost) co-rotation of the vortices, with the superfluid and the crust. The assumed increase in the angular velocity of the crust is shared with the core superfluid over its dynamical timescale $\tau_{\mathrm{D}}=\tau_{\text {free }} . \mathbf{b}$ ) and $\mathbf{c}$ ) are both for a pinned superfluid. Due to the assumed pinning with flux lines, in the initial spinning-down steady-state, a rotational lag between the superfluid and the vortices is necessary. For the "small" jumps, as in b), only the vortices are spun-up over an "azimuthal" relaxation timescale $T_{\mathrm{P} \phi}=T_{\mathrm{P}}$. For the "large" jumps, as in c), the pinned core superfluid couples to the crust over its corresponding dynamical timescale $\tau_{\mathrm{D}}=\tau_{\text {pin }}$, as discussed in the text. 


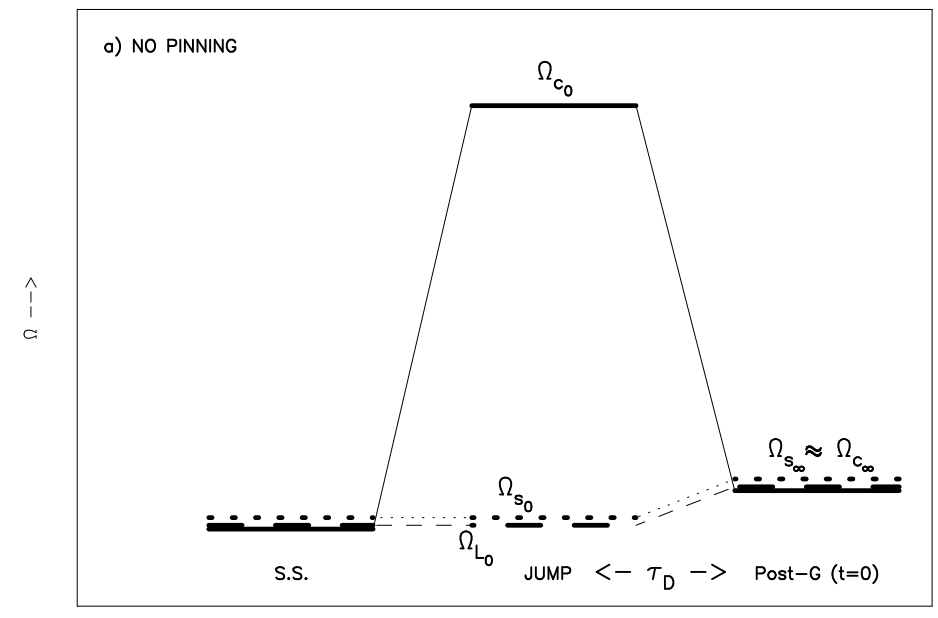

time -->
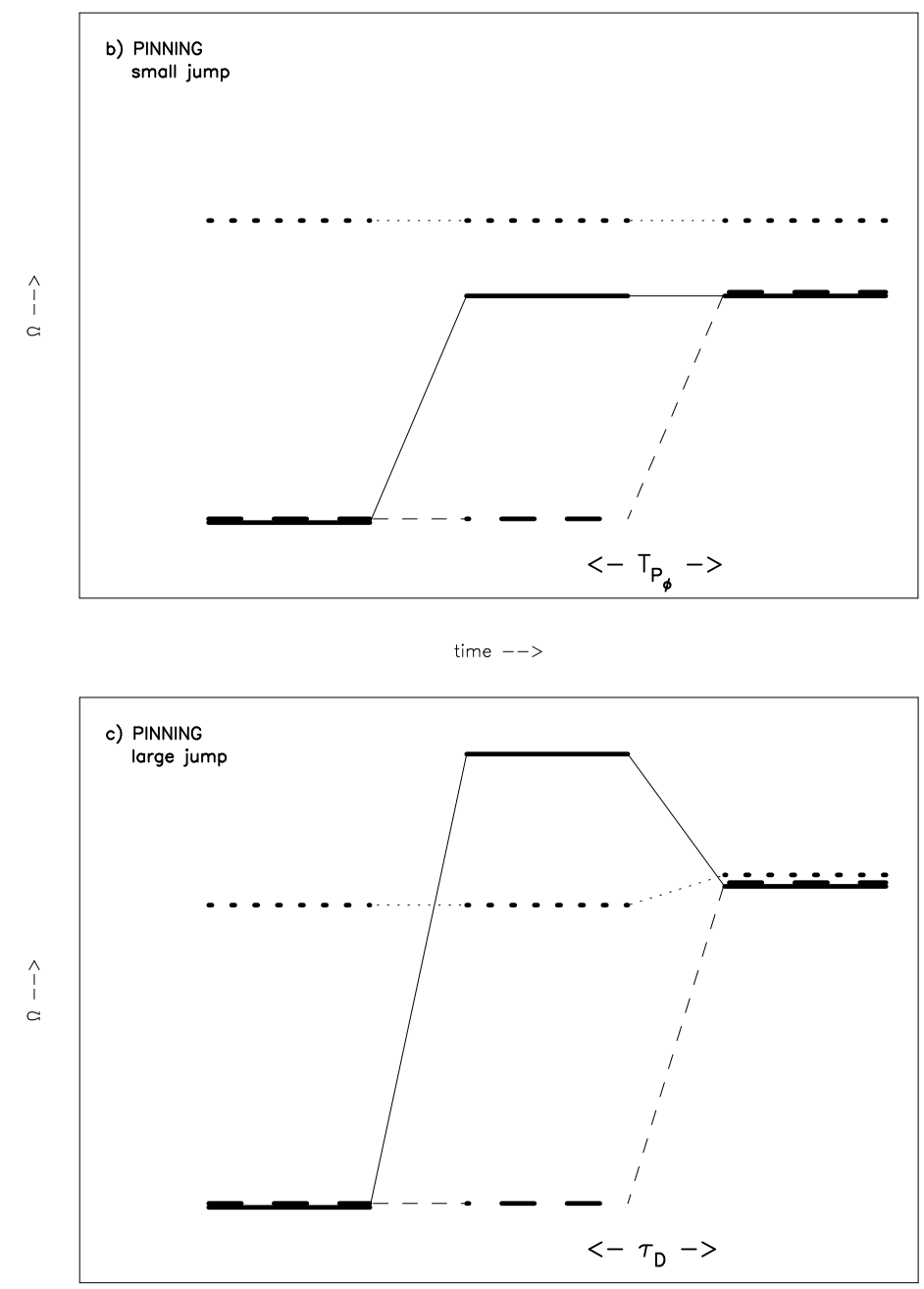

time $-->$ 